ORIGINAL ARTICLE

\title{
Biomechanics of the head for Olympic boxer punches to the face
}

\author{
T J Walilko, D C Viano, C A Bir
}

Br J Sports Med 2005;39:710-719. doi: 10.1136/bjsm.2004.014126

\begin{abstract}
See end of article for authors' affiliations

.....................

Correspondence to:

David C Viano,

ProBiomechanics LLC, 265

Warrington Rd, Bloomfield

Hills, Ml 48304-2952,
\end{abstract}

USA; dviano@comcast.net

Accepted 31 January 2005
Objective: The biomechanics of the head for punches to the jaw and the risk of head injury from translational and rotational acceleration were studied.

Methods: Seven Olympic boxers from five weight classes delivered 18 straight punches to the frangible face of the Hybrid III dummy. Translational and rotational head acceleration, neck responses, and jaw pressure distribution were measured. High speed video recorded each blow and was used to determine punch velocity. Equilibrium was used to determine punch force, energy transfer, and power.

Results: Punch force averaged 3427 (standard deviation (SD) 811 ) N, hand velocity 9.14 (SD 2.06) m/s, and effective punch mass 2.9 (SD 2.0) kg. Punch force was higher for the heavier weight classes, due primarily to a higher effective mass of the punch. Jaw load was 876 (SD 288) N. The peak translational acceleration was 58 (SD 13) g, rotational acceleration was 6343 (SD 1789) rad $/ \mathrm{s}^{2}$, and neck shear was 994 (SD 318) N.

Conclusions: Olympic boxers deliver straight punches with high impact velocity and energy transfer. The severity of the punch increases with weight class.
$\mathrm{T}$ he sports of boxing and karate expose athletes to severe head impacts and the risk of brain injury. ${ }^{1}$ In many cases, the athlete is exposed to repeated impacts and injuries. In a 16 year study of injuries to professional boxers in Australia, 107 injuries were reported in 427 fight participations from August 1986 through to August 2001. ${ }^{2}$ The most commonly injured body region was the head and neck (89.9\%). In this body region, injuries to the eye were the most frequent $(45.8 \%)$ followed by concussions $(15.9 \%)$. There was no information on the mechanism or forces that caused the injuries.

The principles of momentum and energy conservation have been used to estimate the force of various punches and to understand what causes head injuries in karate and boxing. Peak punch forces are reported to range from 1666 to $6860 \mathrm{~N}$. ${ }^{3}$ Walker ${ }^{4}$ estimated that a force of $3200 \mathrm{~N}$ is required to break a brick, which is common practice in karate demonstrations. However, in many studies the momentum of the punch was not transferred to an object comparable in mass and biofidelity to the human head and neck, and thus the risk of injury cannot be estimated from these punches.

In a study of karate, Smith and Hamill ${ }^{5}$ measured the fist velocities from punchers of different skill levels and the relative momentum of a $33 \mathrm{~kg}$ punching bag. Punches to the bag with bare fists (BF), karate gloves (KG), and boxing gloves (BG) were recorded with high speed film. The mean bag momentum for all tests was $47.37 \mathrm{Ns}$. The results showed no significant differences in fist velocities between skill levels or glove type (BF: 11.03 (standard deviation (SD) 1.96) m/s, KG: 11.89 (SD 2.10) m/s, BG: 11.57 (SD 3.43) m/ $\mathrm{s})$. The average fist velocity was $11.5 \mathrm{~m} / \mathrm{s}$. Differences in bag momentum were found with changes in skill level and glove. Greater bag momentum was generated with boxing gloves (53.73 (SD 15.35) Ns) than with either bare fists (46.4 (SD 17.40) Ns) or karate gloves (42.0 (SD 18.7) Ns), which had approximately the same momentum. The bag momentum was also greatest for the highest skilled subjects (60.8 (SD 17.3) Ns) compared to the lower skilled punchers (42.3 (SD 11.6) Ns) even though their respective fist velocities were approximately the same. The authors hypothesised that the increase in bag momentum was due to the skilled boxer's ability to generate a greater effective mass during the impact than the lower skilled boxers. With a fist velocity at $11.5 \mathrm{~m} / \mathrm{s}$ immediately before impact and the resultant bag momentum of $47.4 \mathrm{Ns}$, the effective mass of the striking fist was estimated to be approximately $4.1 \mathrm{~kg}$. This is greater than the mass of the hand and reflects the ability to link more of the arm mass into the punch.

Atha et $a l^{6}$ collected punch force data on a world ranked heavyweight boxer using an instrumented target suspended as a ballistic pendulum. The target was a cylindrical metal mass of $7 \mathrm{~kg}$, estimated to be the mass of the head and neck of a heavyweight boxer. During the punch, the boxer's fist reached an $8.9 \mathrm{~m} / \mathrm{s}$ impact velocity with a resulting peak impact force of $4096 \mathrm{~N}$. The peak acceleration of the pendulum was $53 \mathrm{~g}$. Only one boxer participated in the study so extrapolations to the general boxing population are not possible. However, the results reflect the force of a heavyweight boxer's punch. The model's biofidelity is unknown, so the risk of injury can not be determined. Other studies on punch force have reported peak loads of 4800 (SD 601) N for elite, 3722 (SD 375) $\mathrm{N}$ for intermediate, and 2381 (SD 328) N for novice English boxers ${ }^{7}$ and $3453 \mathrm{~N}$ for 24 elite, $3023 \mathrm{~N}$ for 23 national, and $2932 \mathrm{~N}$ for 23 intermediate boxers. ${ }^{8}$

Smith et al ${ }^{9}$ evaluated the punch of three amateur boxers to assess head impact responses and the risk of injury. Each boxer was instructed to strike a headform with a left hook or left jab. The headform was instrumented with a 3-2-2-2 configuration of accelerometers ${ }^{10}$ to determine the translational and rotational acceleration. The translational acceleration averaged 21.5 (SD 4.6) $g$ for the left jab and 43.6 (SD 15.6) $g$ for the left hook. The rotational acceleration varied from 292.7 (SD 72.2) rad $/ \mathrm{s}^{2}$ for the left jab to 675.9 (SD 230.6) $\mathrm{rad} / \mathrm{s}^{2}$ for the left hook. Based on the tolerance limit of $200 \mathrm{~g}$ for translational acceleration and $4500 \mathrm{rad} / \mathrm{s}^{2}$ for

Abbreviations: BG, boxing gloves; BF, bare fists; DAl, diffuse axonal injuries; $\mathrm{cg}$, centre of gravity; HIC, head injury criterion; KG, karate gloves; MTBI, mild traumatic brain injury; NFL, National Football League; SD, standard deviation 
rotational acceleration, the researchers concluded that neither the translational nor the rotational acceleration reached a level that was injurious to the boxer. They suggested that repeated sub-concussive blows were the injury mechanism for mild traumatic brain injury (MTBI).

In contrast, Johnson et $a l^{11}$ reported that head injuries are likely in boxing. Low velocity volunteer data were extrapolated to predict higher impact velocities that were more representative of those seen in boxing. The report determined that the extrapolated data fell in the region of "certain injury" calculated by Unterharnscheidt and Sellier. ${ }^{12}$ Their study assumed that the maximum punch strength was delivered to the head with only minimal deflection. While the results of this study raise concerns about the risk of injury in boxing, the use of extrapolated low speed data to predict injury at higher speeds needs additional validation.

Techniques have been developed to determine the risk of head injury for an impact. ${ }^{13}$ Based on biomechanical principles, these techniques involve the simulation of realworld impacts on human surrogates with biofidelity built into their impact responses. ${ }^{14}$ Biofidelity reflects the ability of the surrogate to simulate the essential biomechanical characteristics of the human impact response. The Hybrid III crash dummy used in this project is currently the most advanced, validated biomechanical surrogate for frontal impacts. The validation of the surrogate includes the head and neck, which are of particular interest in this study. The head and neck of the Hybrid III are used in many state of the art side impact dummies, so its use includes a range of impact angles. Sensors placed in the surrogate collect biomechanical data that can be related to risk of injury. Previous studies have developed a collection of criteria to estimate the risk of injury from impacts. ${ }^{15-17}$

The techniques used to estimate the risk of head injury are based on relating measured responses to observed injuries. The first to establish a relationship between head injury and translational acceleration was Lissner. ${ }^{18}$ His observations led researchers to develop a curve relating the level of acceleration and the duration of the impact to the risk of head injury. ${ }^{13}{ }^{19}$ Based on these initial observations, it is currently believed that impacts to the head that have low peak acceleration require longer pulse durations to cause the same injuries as those with high peak acceleration. The head injury criterion (HIC) currently used to assess risks is calculated as follows:

$$
\mathrm{HIC}=\left\{\left(\mathrm{t}_{2}-\mathrm{t}_{1}\right)\left[\int_{\mathrm{t}_{1}}^{\mathrm{t}_{2}} \mathrm{a}(\mathrm{t}) \mathrm{dt} /\left(\mathrm{t}_{2}-\mathrm{t}_{1}\right)\right]^{2.5}\right\}_{\max }
$$

where $t_{1}$ and $t_{2}$ are determined to give the maximum value to the HIC function and $\mathrm{a}(\mathrm{t})$ is the resultant translational acceleration of the head centre of gravity $(\mathrm{cg})$. In practice, a maximum limit of $\mathrm{T}=\mathrm{t}_{2}-\mathrm{t}_{1}=15 \mathrm{~ms}$ is used.

The US delegation to the ISO working group 6 provided an estimate of the percentage of the adult population expected to experience a life threatening brain injury (Abbreviated Injury Scale 4) for various HIC levels due to frontal head impacts. ${ }^{20}$ The delegation's best estimate was that $16 \%$ of the adult population would experience a life threatening brain injury at an HIC level of 1000. In recent studies of concussions in National Football League (NFL) players in the USA, Pellman $e a^{21}{ }^{22}$ recommended a value below 250 to minimise the risk of MTBI or concussion.

The British physicist Holbourn ${ }^{23}$ worked with gel models of the brain and was one of the first to cite rotational acceleration as an important mechanism in head injury.
Ommaya and Hirsch ${ }^{24}$ scaled primate head injury data to humans and predicted that a level of head rotational acceleration in excess of $1800 \mathrm{rad} / \mathrm{s}^{2}$ would have a $50 \%$ probability of resulting in cerebral concussion in man. Analysis of injuries produced in rhesus monkey experiments resulted in Gennarelli et al ${ }^{25}$ estimating a $16000 \mathrm{rad} / \mathrm{s}^{2}$ rotational acceleration tolerance threshold in man.

In a survey of their research on rotation head injury, Ommaya et $a l^{17}$ stated that for the adult brain the rotational acceleration required to produce concussion is $4500 \mathrm{rad} / \mathrm{s}^{2}$ and severe diffuse axonal injuries (DAI) occur at rotational accelerations of $18000 \mathrm{rad} / \mathrm{s}^{2}$. This response range is obtained from scaling animal impact data and indicates the difficulty in developing a precise injury prediction criterion for rotational motion. One problem is that the shape and mass of the animal and human brains are different and scaling laws assume geometric similarity. The low mass of the animal brain requires very high rotational accelerations to produce closed head injuries. ${ }^{24}$ These factors complicate predictions of human injury from animal responses to rotational head acceleration.

In an effort to understand the relationship between forces delivered to the jaw region and the risk of head injury from translational and rotational acceleration, the biomechanics of boxer's punches were studied. Olympic class boxers threw straight punches at an instrumented Hybrid III headform with their dominant hand. Punch force, weight class, and the severity of head translational and rotational acceleration were correlated.

\section{METHODS}

\section{Test set up}

Ten Olympic boxers participated in the study, but only data from seven were suitable for analysis and inclusion. Each boxer delivered three punches that were evaluated for strength and severity. The weight of the boxers ranged from $112 \mathrm{lbs}(48 \mathrm{~kg})$ to $240 \mathrm{lbs}(109 \mathrm{~kg}$ ) and represented four weight classes. After each boxer had warmed up, they were instructed to strike the lower third of the headform on the instrumented dummy with their gloved fist three times using maximum effort. The exact impact location of the punch was determined and translational force, hand velocity, and effective mass were calculated. The severity of the impacts was quantified using rotational acceleration, HIC, and head velocity.

Because a frangible face is used, ${ }^{26}$ the dummy's impact response is similar to that of a human, so the risk of injury to the boxer's hand was no greater than the risk during normal training. However, although the risk of injury was minimal, a certified boxing trainer was present throughout the testing and informed written consent was obtained from each boxer. Since their involvement in the study was voluntary, it was made clear that the boxers could withdraw from the study at any time. The boxers were not compensated for their participation. Approval for the study was obtained through Wayne State University's Human Investigation Committee.

A Hybrid III dummy with a frangible faceform was used to represent the response of the jaw and realistically transfer acceleration to the head. Figure 1 (right) shows the side view of the frangible face and Hybrid III headform. ${ }^{26}$ It has an improved biomechanical response in the facial region over the standard moulded Hybrid III and is capable of more accurately reproducing the force and acceleration measurements of the head for impacts in the frontal, zygomatic, maxillary, and mandibular regions. Other devices have used either a stiff load measuring face or deformable structures in regions other than the jaw. ${ }^{28}$ The frangible Hybrid III dummy headform was attached to the Hybrid III neck and upper torso to ensure realistic headform motion (fig l, left). 

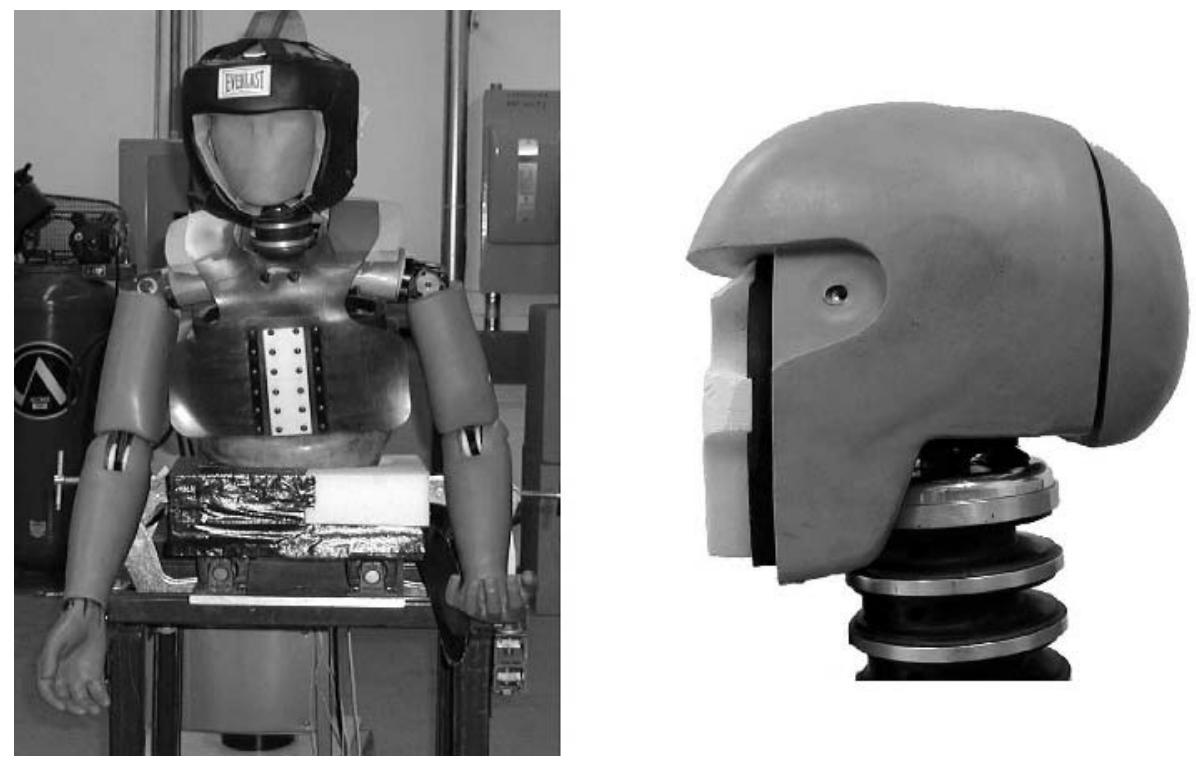

Figure 1 Test fixture with Hybrid III dummy attached. Image on the right is a breakout of the Hybrid III headform with frangible face foam subassembly exposed.

Olympic headgear was placed on the dummy, but the blows were straight to the face and did not engage the headgear. For positioning, the upper torso was attached to a rigid table with a foam pad placed below the Hybrid III abdomen insert so that the dummy remained in an upright position during the impact.

The Hybrid III simulates a tensed neck so the head is normally upright. The segmented neck includes flexible polymer discs to simulate the flexion-extension and lateral bending responses. A cable inside tightens the assembly to give the correct neck response in calibration testing and during head acceleration..$^{29}{ }^{30}$ Although the Hybrid III neck was utilised in the study, it is not known if it accurately represents the strength of a boxer's neck since a boxer undergoes extensive training to develop the neck muscles necessary to resist the force delivered by an opponent. Johnson et $a l^{11}$ demonstrated that neck muscle tension has little effect on the oscillation of the head under sinusoidal excitation from a shaker.

\section{Instrumentation}

Accelerometers were placed in the boxer's hand and in the head of a Hybrid III dummy to measure the impact dynamics. Seven Endevco (San Juan Capistrano, CA) 7264-2000 accelerometers were used in the evaluation; two were secured to the boxer's hand to measure overall hand dynamics, while the remaining five were fastened to a bar within the headform for calculation of translational and rotational acceleration using the in-line technique. ${ }^{31} 32$ Figure 2 shows the two accelerometers attached to a magnesium block that was tightly taped to the clenched fist to measure hand dynamics. The two accelerometers were at right angles so that the resultant hand acceleration could be measured. A third accelerometer was not included because it was assumed that the lateral acceleration of the hand was negligible for a straight punch. The block was secured to the boxer's hand by embedding it in the boxer's hand wrap. This fixation method did not rigidly attach the accelerometers to the hand but did provide a good method of predicting the overall hand dynamics.

A six axis upper neck load cell (Denton ATD, Rochester Hills, MI) was used to measure neck loads and moments. An event switch was attached to the face of the dummy and determined initial glove/headform contact giving a precise initiation signal establishing time zero. The primary data acquisition system was the IDDAS data acquisition system (SoMat, Urbana, IL). Data were collected at a sampling rate of $14.7 \mathrm{kHz}$ and post processed according to SAE J21 $1 / 1 .{ }^{33}$

\section{Tekscan pressure sensing system}

A Tekscan pressure sensor (Boston, MA) was inserted between the frangible faceform and the headform skin to measure the force on the jaw region. The Tekscan pressure system measures the pressure and contact area of a punch applied to any section of the sensor. The Tekscan sensor (Model 9500) used was an extremely thin $(\sim 0.1 \mathrm{~mm})$, flexible tactile pressure sensor capable of measuring pressures ranging from 0 to 2000 psi. The size of the sensing surface was $71 \times 71 \mathrm{~mm}$ with a resolution of 3.9 sensors per centimetre. The sampling rate of the system was $1.4 \mathrm{kHz}$. The

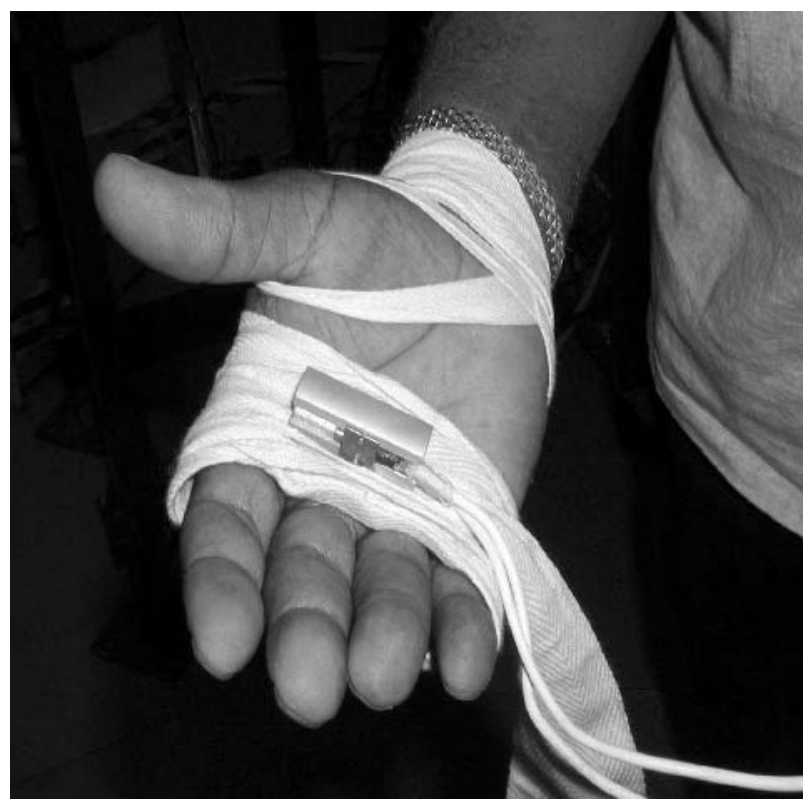

Figure 2 Accelerometer package inserted into his handwrap and used to measure the boxer's hand motion. Photograph reproduced with patient consent. 
output from the system is the pressure distribution on the surface of the sensor and the corresponding contact area as a function of time. The force on any region is calculated by multiplying the sum of the individual pressure measurements within the selected region by the active contact area. The Tekscan and IDDAS systems were synchronised with a common start trigger so that the data from both systems could be aligned for the post processing analysis.

\section{Video film analysis plus target location}

Targets were placed on the lateral surface of the glove near the junction between the hand and wrist to digitise its motion and calculate impact velocity. Additional targets were attached to the head and the spine of the Hybrid III to measure the overall kinematics of the dummy during impact. Images were captured with a Kodak HG2000 high speed video camera. The camera recorded the event at 1000 images per second. Digitisation of the data was completed using Image Express for video recording and processed according to SAE J $211 / 2 .^{34}$

\section{Data collection procedure}

After an appropriate warm up period, the boxer was asked to lightly punch the head of the instrumented dummy with their wrapped and gloved hand. If there was no pain or discomfort, they were asked to increase their punch strength until they reached a point where they were throwing "normal" punches. Once the boxer was comfortable throwing punches, they were instructed to strike the instrumented dummy three times with a straight punch to the jaw region with their dominant hand. Figure 3 shows the orientation of the punch. Both left and right handed boxers participated in this study. In the final analysis there was no way of determining if the boxer was delivering his maximal punch force, but the competition that developed between the boxers led the researchers to believe that they were delivering punches with maximal effort.

\section{Calculation of head translational and rotational acceleration}

Rotational acceleration of the headform was calculated using the $2 \mathrm{D}$ in-line method. ${ }^{31}{ }^{32}$ This method takes into account differences in acceleration measured on a rigid body to derive rotational acceleration about an axis. Translational accelerations ( $\mathrm{x}$ and $\mathrm{z}$ direction) were measured to calculate the rotational acceleration about the y axis. Axis transformation

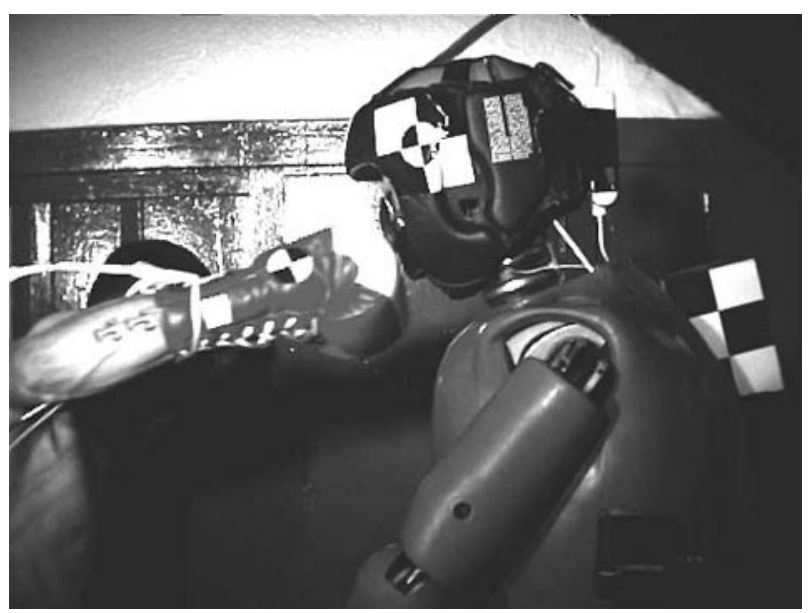

Figure 3 Olympic boxer throwing straight right punch to jaw region of instrumented Hybrid III dummy. equations were used to transfer the measured and calculated accelerations to the headform $\mathrm{cg}$.

\section{Calculation of punch force}

Figure 4 shows the $\mathrm{x}$ direction equilibrium of forces during a boxer's punch. The equilibrium was determined using the upper neck shear load and headform $\mathrm{x}$ direction $\mathrm{cg}$ acceleration. Summing forces in the $\mathrm{x}$ direction gives the mass times head acceleration:

$$
\mathrm{Fp}_{\mathrm{x}}-\mathrm{Fn}_{\mathrm{x}}=\mathrm{mA}_{\mathrm{x}}
$$

where $\mathrm{Fp}_{\mathrm{x}}$ is the force applied to the headform by the boxer in the $\mathrm{x}$ direction, $\mathrm{Fn}_{\mathrm{x}}$ is the neck shear force, $\mathrm{m}$ is the mass of the headform, and $A_{x}$ is the $x$ direction translation acceleration at the headform cg. This relationship assumes the motion of the headform does not substantially alter the orientation of the head and neck, so the lateral and vertical responses become important. These effects occur later in the impact. By measuring the neck shear force and head acceleration, the punch force can be determined from eq 2 .

\section{Effective mass of the punch}

The effective mass of the boxer's punch was determined using the momentum equation:

$$
\mathrm{m}_{\mathrm{h}} \mathrm{V}_{\mathrm{p}}=\left(\mathrm{m}_{\mathrm{h}}+\mathrm{m}\right) \mathrm{V}_{\mathrm{h}}
$$

where $m_{h}$ is the effective mass of the puncher's hand, $V_{p}$ is the punch velocity, $\mathrm{V}_{\mathrm{h}}$ is the Hybrid III head velocity, and $\mathrm{m}$ is the mass of the Hybrid III head. The Hybrid III head weighs $3.64 \mathrm{~kg}$ for components above the upper neck load cell. This equation neglects the portion of the neck and torso involved in the impact. These masses are influential later in the impact sequence. The peak velocity of the boxer's hand was derived from integration of the resultant hand acceleration. This value was confirmed by differentiation of the hand motion collected by high speed video. Translational head velocity was calculated by integrating the translational acceleration of the head cg.

\section{Calculation of punch energy transfer and power}

Energy transfer to the head was determined by multiplying the Hybrid III head mass by the square of the head velocity determined by integration of the $\mathrm{x}$ direction acceleration of the Hybrid III head. The punch power was determined as the time varying product of the punch force times the Hybrid III head velocity.

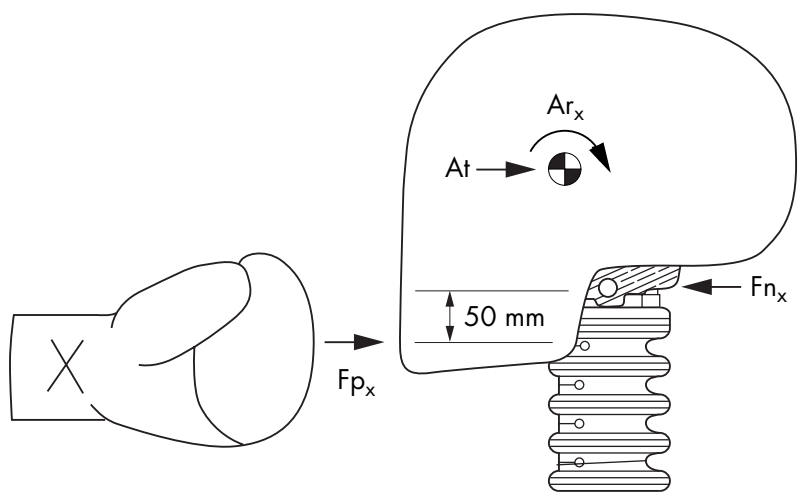

Figure 4 Equilibrium of forces used to determine punch force in the $x$ direction. 


\section{RESULTS}

\section{Evaluation of Olympic boxers}

Thirty punches from 10 boxers were analysed. The punches were classified as either straight, extended, or glancing blows. Ten of the 30 punches (33\%) were classified as a straight punch defined as a punch where the wrist remained rigid and straight. Eight (27\%) were extended punches where the wrist dorsiflexed shortly after impact. The third impact condition was the glancing blow where the blow did not land cleanly but rolled off the headform to the right or left (40\%). The glancing blows were neglected in the analysis. In the final analysis, 18 punches from four weight classes and seven boxers (flyweight, light welterweight, middleweight, and super heavyweight) were used.

Calculated punch force and biomechanical responses Table 1 shows that the peak punch force ranged from 1990 to $4741 \mathrm{~N}$. The mean force for all 18 landed punches was 3427 (SD 811) N. Using the one way ANOVA procedure, an analysis of variance for punch force was conducted for wrist position. There was no significant difference $(p=0.16)$ between the rigid wrist (367l (SD 814) N) and the flexed wrist (2775 (SD 780) N) in terms of mean punch force. Peak punch force and weight class were compared using the same procedure. There was a significant difference $(p=0.021)$ in punch force between the various weight classes. The relationship between force and weight was determined by Pearson's correlation, which measures the association between variables. Table 2 shows that punch force increases linearly with weight class $(\mathrm{r}=0.539, \mathrm{p}=0.021)$.

The Tekscan system was used to measure the portion of the impact force delivered to the jaw region, which was defined as the bottom third of the faceform. The study set out to determine the amount of force entering the base of the skull through the mandible. Tekscan was used because in many instances the punch was centred higher on the headform and covered a greater area, so the total force would not represent the force delivered to just the jaw region. The mean force applied to the mandible for all landed punches was 876 (SD 288) $\mathrm{N}$ (table 1). There was no significant difference $(\mathrm{p}=0.077)$ between force applied to the jaw with the rigid (984 (SD 300) N) and the flexed wrist (616 (SD 199) N).

The Hybrid III neck absorbed a portion of the overall punch force. The mean neck shear force for all 18 landed punches was 994 (SD 318) N (table 1). There was no significant difference $(p=0.100)$ between neck shear force with the rigid (1105 (SD 386) N) and the flexed wrist (761 (SD 166) $\mathrm{N}$ ). $\mathrm{N}_{\mathrm{ij}}$ (overall neck injury risk value) averaged 0.27 (SD 0.07) and was well below the current tolerance level of $\mathrm{N}_{\mathrm{ij}}=1.0$.

\section{Hand velocity}

The hand velocity was determined by integrating the resultant hand acceleration up to face contact. The mean hand velocity at impact was 9.14 (SD 2.06) m/s (table 1). The glove motion was tracked using video analysis to verify the accuracy of the integrated hand velocity targets. The positiontime traces generated from the analysis were differentiated to obtain hand velocity. The results showed good correlation between the two velocity measurements. Data relating to hand velocity were analysed using integrated hand acceleration.

ANOVA analysis determined that there was a significant difference $(\mathrm{p}=0.019)$ between the rigid wrist $(8.16$ (SD $1.38) \mathrm{m} / \mathrm{s}$ ) and the flexed wrist (9.21 (SD 2.17) $\mathrm{m} / \mathrm{s}$ ) in terms of hand velocity. Comparisons between hand velocity and weight class showed no significant difference, but the association between the two variables is not linear $(\mathrm{r}=-0.071, \mathrm{p}=0.779$; table 2$)$.
Effective mass of the punch

The mean effective mass for all landed punches was 2.86 (SD $2.03) \mathrm{kg}$. No significant differences were seen between rigid and flexed wrist groups $(\mathrm{p}=0.271)$. The analysis also showed the effective mass varies with translational force $(p=0.012)$ but only has a slight linear association with the weight of the boxer $(\mathrm{r}=0.484, \mathrm{p}=0.042)$.

\section{Resultant head acceleration}

The resultant translational headform acceleration was determined using the calculated accelerations from the 2D in-line method. Prior to calculating the rotational accelerations, the translational acceleration traces were pre-processed using a $1650 \mathrm{~Hz}$ Butterworth filter specified in the SAE J211 standard. The mean head acceleration for the headform $\mathrm{cg}$ was 58 (SD 13) $\mathrm{g}$ with average duration of $11.4 \mathrm{~ms}$. The analysis of variance determined that there was no significant difference $(p=0.135)$ between the rigid wrist and the flexed wrist with respect to translational acceleration of the headform.

Comparisons between acceleration and weight class were made using the same procedure. Pearson's correlation coefficient was calculated to determine the relationship between translational acceleration and boxer weight. A weak linear relationship existed between the two variables $(\mathrm{r}=0.432, \mathrm{p}=0.074)$. The final translational velocity of the head was determined by integrating the head cg acceleration in the $\mathrm{x}$ direction. The average $\Delta \mathrm{V}$ (change in head velocity) of the head was 2.97 (SD 0.81 ) $\mathrm{m} / \mathrm{s}$. Comparisons between head velocity and weight class showed a significant difference $(p=0.017)$ with respect to the boxer's weight (table 1 ) and the association between the two variables was linear $(\mathrm{r}=0.555, \mathrm{p}=0.085$, table 2$)$.

The rotational acceleration of the headform about the $y$ axis was calculated using the $2 \mathrm{D}$ in-line method. The mean rotational head acceleration for all landed punches was 6343 (SD 1789) $\mathrm{rad} / \mathrm{s}^{2}$ (table 1 ). The analysis of variance determined that there was no significant difference $(p=0.423)$ between the rigid wrist and the flexed wrist with respect to rotational acceleration of the headform (table 1). However, a linear relationship existed between weight and rotational acceleration $(\mathrm{r}=0.524, \mathrm{p}=0.026$; table 2$)$.

Statistical analysis of the injury parameter HIC showed that there was a significant difference $(p=0.071)$ between the rigid wrist (90 (SD 53)) and the flexed wrist (43 (SD 37)) groups. The mean HIC from all punches was 71 (SD 49). The ANOVA revealed that HIC varies with weight class $(p=0.002)$. The Pearson correlation test determined that the relationship is approximately linear $(r=0.672, p=0.002)$ with respect to the boxer's weight. Using the same procedure, it was determined that HIC also has a linear relationship with punch force $(\mathrm{r}=0.886, \mathrm{p}=0.000)$.

\section{Kinematic analysis of the headform}

Figure 5 shows the kinematics of a flyweight boxer where the wrist remained rigid (us018-3). Initial contact of the glove with the headform was signalled by an event trigger attached to the headform (fig 5, line and photo A). Peak rotational $\left(5209 \mathrm{rad} / \mathrm{s}^{2}\right)$ and translational $(68 \mathrm{~g})$ acceleration occurred approximately 3 and $4 \mathrm{~ms}$, respectively, after initial contact (fig 5, lines and photos $\mathrm{B}$ and $\mathrm{C}$ ). The total duration of impact was $13 \mathrm{~ms}$. The peak force of $3737 \mathrm{~N}$ occurred $5 \mathrm{~ms}$ after initial contact. The peak force applied to the jaw was $1047 \mathrm{~N}$ and the peak neck shear force was $946 \mathrm{~N}$. Both occurred with the peak punch force. The effective mass for this punch was $4.4 \mathrm{~kg}$ with a corresponding hand velocity of $6.7 \mathrm{~m} / \mathrm{s}$. The calculated HIC was 101. The end of the blow occurred at line $\mathrm{E}$ in the graph, when the fist contacted the chest of the Hybrid III dummy. 


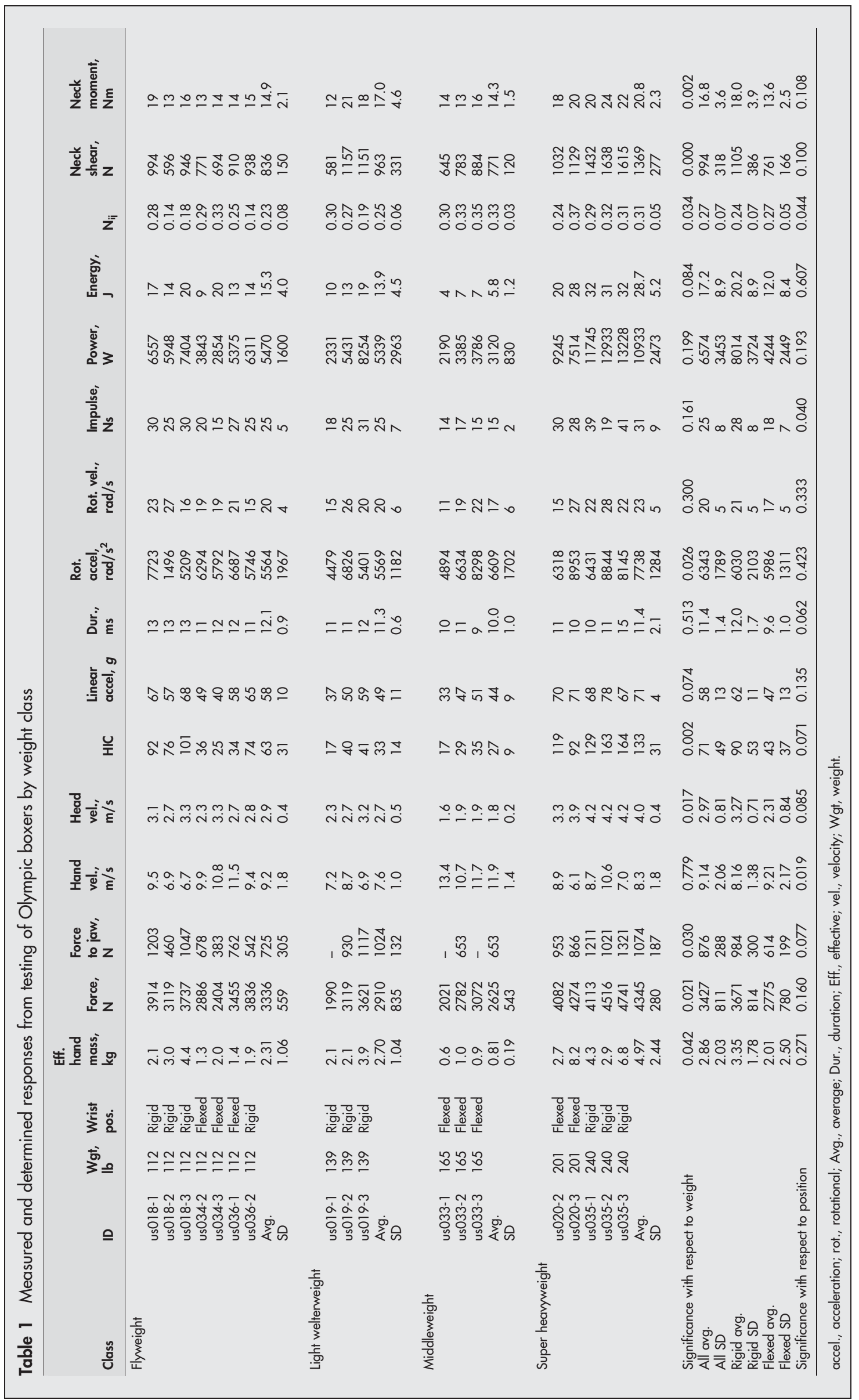


Table 2 Correlation between punch dynamics and risk of injury parameters

\begin{tabular}{|c|c|c|c|c|c|c|c|c|c|}
\hline & Weight & $\begin{array}{l}\text { Punch } \\
\text { force }\end{array}$ & $\begin{array}{l}\text { Hand } \\
\text { velocity }\end{array}$ & $\begin{array}{l}\text { Effective } \\
\text { mass }\end{array}$ & $\begin{array}{l}\text { Force } \\
\text { to jawt }\end{array}$ & HIC & $\begin{array}{l}\text { Linear } \\
\text { acceleration }\end{array}$ & $\begin{array}{l}\text { Head } \\
\text { velocity }\end{array}$ & $\begin{array}{l}\text { Rotational } \\
\text { acceleration }\end{array}$ \\
\hline Weight & - & $0.539 *$ & -0.071 & $0.484^{*}$ & $0.561^{*}$ & $0.672^{* *}$ & 0.432 & $0.555^{*}$ & $0.524^{*}$ \\
\hline Punch force & $0.539 *$ & - & -0.400 & $0.661^{\text {** }}$ & $0.736^{* *}$ & $0.886^{* *}$ & $0.961^{\text {** }}$ & $0.806^{* *}$ & $0.528^{*}$ \\
\hline Hand velocity & -0.071 & -0.400 & - & -0.758 & -0.348 & -0.369 & -0.416 & $-0.519^{*}$ & 0.170 \\
\hline Effective mass & $0.484^{*}$ & $0.661^{*}$ & $-0.758^{*}$ & - & 0.476 & $0.618^{*}$ & $0.589^{*}$ & $0.774^{*}$ & 0.280 \\
\hline Force to jawt & $0.561^{*}$ & $0.736^{* *}$ & -0.348 & 0.476 & - & $0.631^{* *}$ & $0.621^{* *}$ & $0.580^{*}$ & 0.500 \\
\hline $\mathrm{HIC}$ & $0.672^{* *}$ & $0.886^{* *}$ & -0.369 & $0.618^{* *}$ & $0.631^{* *}$ & - & $0.862^{* *}$ & $0.829 * *$ & 0.372 \\
\hline Linear acceleration & 0.432 & $0.961^{\text {** }}$ & -0.416 & $0.589^{* *}$ & $0.621^{\text {** }}$ & $0.862^{* *}$ & - & $0.769^{* *}$ & 0.425 \\
\hline Head velocity & $0.555^{*}$ & $0.806^{* *}$ & $-0.519^{*}$ & $0.774^{\star *}$ & $0.580^{*}$ & $0.829^{* *}$ & $0.769^{* *}$ & - & 0.361 \\
\hline Rotational acceleration & $0.524^{*}$ & $0.528^{*}$ & 0.170 & 0.280 & 0.500 & 0.372 & 0.425 & 0.361 & - \\
\hline
\end{tabular}

\section{Punch energy transfer and power}

The energy transfer from all punches averaged 17.2 (SD 8.9) $\mathrm{J}$ (or Nm). The punch power averaged 6574 (SD 3453) W ( or $\mathrm{J} / \mathrm{s}$ ). Both values increased when only the rigid wrist tests were averaged to 20.2 (SD 8.9) J energy transfer and 8014 (SD 3724) W punch power.

\section{DISCUSSION}

The current study contributes new information about head injury risks by combining data on the head impact response of a biofidelic dummy and the measured punch force of Olympic class boxers throwing straight punches. The headneck assembly of the Hybrid III headform closely represents the mass and compliance of the average human head and neck. With this system, both the risk of injury in terms of HIC, and translational and rotational acceleration were explored as regards the momentum transfer by a punch to a biofidelic Hybrid III surrogate.

The average peak force was 2625 (SD 543) $\mathrm{N}$ for the middleweight boxer and 4345 (SD 280) $\mathrm{N}$ for the super heavyweights. This is consistent with the peak forces reported in the literature which range from 1666 to $6860 \mathrm{~N}^{3}{ }^{6-8}$ The boxers' hand velocity was 9.14 (SD $2.06) \mathrm{m} / \mathrm{s}$ and had no significant correlation with the weight of the boxer, peak force, or severity of the punch. This level is lower than that determined by Smith and Hamill ${ }^{5}$ and may reflect the fact that these tests only involved straight punches. A later study by Smith et $\mathrm{al}^{7}$ found peak punch forces in the range of values reported here.

Statistical analysis of the injury parameter HIC showed there was a significant difference $(p=0.06)$ between the rigid wrist (90 (SD 51)) and the flexed wrist (48 (SD 37)) groups. The mean HIC from all punches was 71 (SD 48). The ANOVA revealed that HIC varies with weight class $(p=0.0005)$. The Pearson correlation test determined that the relationship is approximately linear $(r=0.672, p=0.002)$ with respect to the boxer's weight. The same procedure showed that HIC also has a linear relationship with punch force $(r=0.889, p=0.000)$. These findings indicate that the differences in HICs are better explained by changes in the punch force generated than by the weight of the boxer. However, the relationship between wrist position and risk of injury is unknown at this time. In both positions, the risk of injury predicted by HIC is less than $2 \%$ implying a very low risk of injury from translational acceleration.

High rotational accelerations were calculated. The average peak rotational acceleration was 6343 (SD 1789) rad/s $\mathrm{s}^{2}$. Ommaya et $a l^{17}$ indicated a rotational acceleration of approximately $4500 \mathrm{rad} / \mathrm{s}^{2}$ was required to produce concussion. They also stated that severe DAI occurred at $18000 \mathrm{rad} / \mathrm{s}^{2}$, and moderate and mild DAI at 15500 and $12500 \mathrm{rad} / \mathrm{s}^{2}$, respectively. Earlier studies by Pincemaille et $a l^{35}$ measured rotational accelerations of $13600 \mathrm{krad} / \mathrm{s}^{2}$ and rotational velocities of $48 \mathrm{rad} / \mathrm{s}$ during boxing; no cases of concussion were reported. The current tests with the Hybrid III also show high rotational accelerations, however, the data reflect a higher tolerance than those specified by Ommaya et $a l^{17}$ and Gennerelli et al..$^{25}$ Since their tolerances are based on scaling of animal data, a question may be raised about the adequacy of the technique, which assumes similar geometry and equivalent material characteristics between animal and man.

In recent studies of concussion in professional football, Pellman et $a^{21}{ }^{22}$ reproduced game impacts with Hybrid III dummies and found concussion occurred at average peak rotational accelerations of 6596 (SD 1866) rad $/ \mathrm{s}^{2}$. These levels are consistent with the rotational accelerations of 6343 (SD 1789) $\mathrm{rad} / \mathrm{s}^{2}$ found in this study. However, the strongest correlations with concussion were with translational acceleration and head $\Delta \mathrm{V}$. No concussions were found with peak accelerations of 68 (SD 15) $g$ and HIC of 143 (SD 37). These levels are above that determined for the boxing punch. In the NFL, concussion occurred with head accelerations of 94 (SD 28) $g$ and HIC of 345 (SD 181). These levels are well above those delivered by the Olympic boxers to the Hybrid III dummy. Based on this work, it can be hypothesised that rotational acceleration is an important factor in boxing injuries. Using the injury risk functions given in Pellman et $a l^{21}$ the risk of concussion for the boxer punches averaged $13 \%$ (SD 10\%) for HIC, 20\% (SD 2\%) for translational head acceleration, and 3\% (SD 0\%) for head $\Delta \mathrm{V}$, but $68 \%$ (SD 3\%) for rotational acceleration.

Rotational acceleration had good linear correlation with weight class. Weight class also showed good correlation with punch force, jaw force, HIC, and head velocity. These results support previous epidemiological studies showing that head injuries occur more frequently in the heavier weight classes ${ }^{36}$ and the general mechanics of the boxing punch. ${ }^{37}$ While weight was a good predictor, punch force had a stronger correlation with HIC, rotational acceleration, and head velocity. In addition, punch force also correlated with translational acceleration. Hand velocity did not seem to affect the severity of impact. This means the effective mass of the boxer's punch is more important in increasing the severity of a blow.

The effective mass from the punch was determined using conservation of momentum. The effective mass for all boxers analysed averaged 2.9 (SD 2.0$) \mathrm{kg}$. The effective mass for the flyweights was 2.3 (SD 1.1) kg, for the light welterweights 2.7 (SD 1.1) kg, for the middleweight 0.8 (SD 0.2 ) $\mathrm{m} / \mathrm{s}$, and for the super heavyweights 5.0 (SD 2.4) kg. The effective mass calculated for the flyweights, light welterweights, and the super heavyweights approximated the effective mass of their arms. ${ }^{38}$ The only middleweight boxer evaluated had a smaller calculated effective mass due to the boxer dorsal flexing his wrist upon impact. In this case, the effective mass was approximately the mass of the middleweight boxer's hand. 

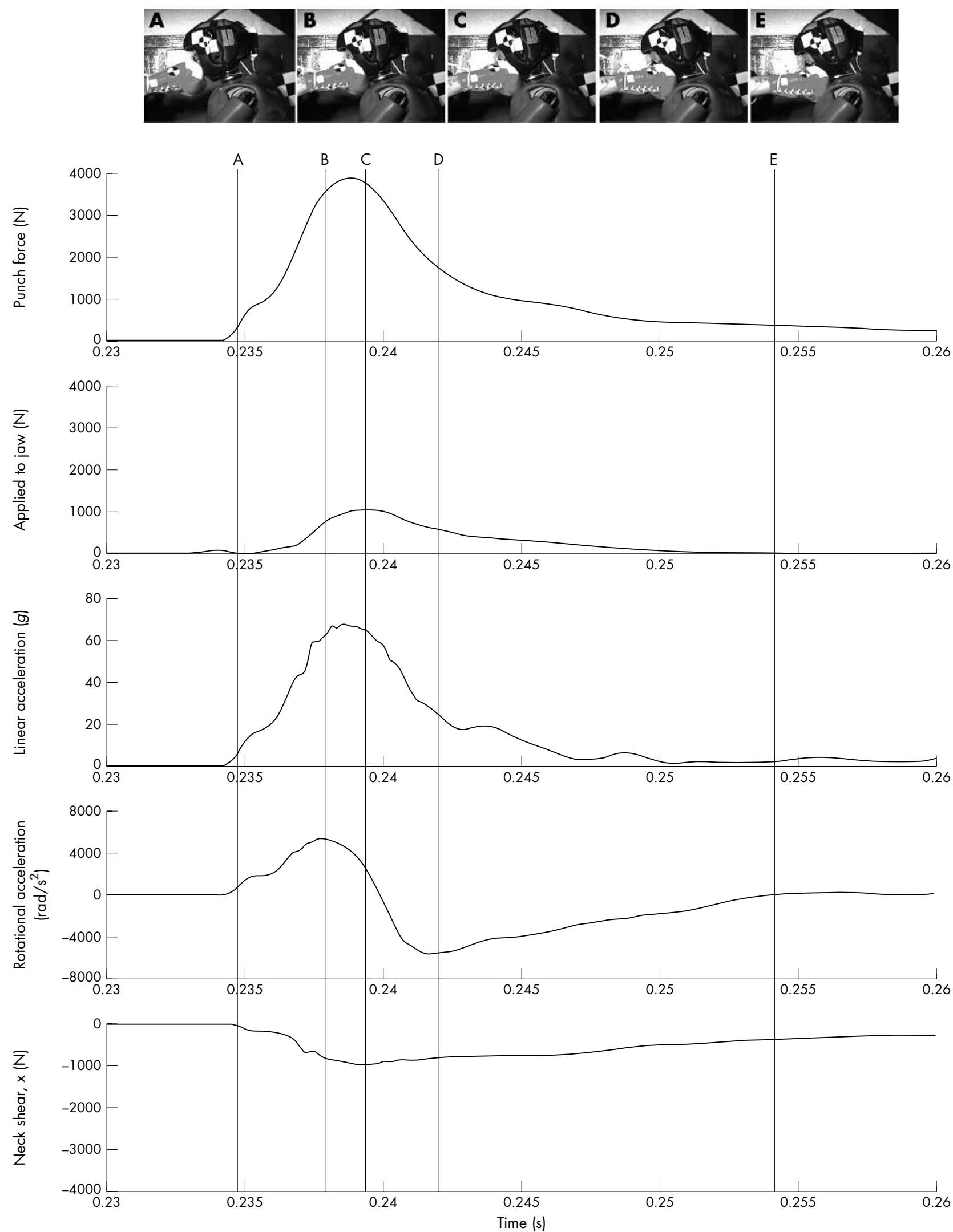

Figure 5 Punch dynamics showing time history of impact responses. 
What is already known on this topic

Previous research has determined the impact force of a boxer's punch.

\section{What this study adds}

This study uses a humanlike, frangible dummy face to determine the punch force to the jaw and head dynamics for Olympic level boxers.

Flexing the wrist decreases the effective mass behind the punch and decreases the risk of injury as estimated by HIC. HICs are higher for the rigid wrist because of the increased duration and the effective mass of the impact. When the wrist flexes, the palm of the hand contacts the chest decreasing the duration of the pulse by $10 \%$. In either scenario, HICs are low for straight blows to the chin with a risk of severe traumatic brain injury of $<2 \%{ }^{20}$ The average HIC in the current study was 71 (SD 49), well below the proposed NFL concussion threshold of $250 .^{21} 22$

\section{Limitations}

While the number of punches collected and analysed in this project was low, the results could help define the acceleration conditions under which MTBI occurs in boxing. There are also possibilities for developing useful training methods to teach boxers techniques to increase the effective mass of their punch. The described methodology of measuring translational and rotational accelerations could be applied to the regular evaluation of punch strength and severity for other punches such as the jab, hook, and uppercut. Data from this study may be useful in developing standards for evaluation of boxing gloves and head gear.

The complexity of quantifying the severity of presumably knockout punches to the jaw region by Olympic class boxers is illustrated by the head acceleration measurements. Low HIC measurements indicate that translational acceleration of the head in this study had only a marginal influence on the severity of a boxer's punch. In contrast, rotational accelerations appeared to be the dominate response within the study and were consistent with levels found in concussion of professional football players..$^{21}$ However, additional research is required to clarify the importance of translational and rotational acceleration of the head for the severity of a punch delivered to the jaw region. The cumulative effect of multiple punches landed on the head was not addressed in this study. The acceleration levels reported in this study may be sufficient to cause some level of head injury if multiple punches are landed. Thus, analysis of additional parameters not collected in this study may be necessary to fully understand the injury mechanism of the knockout punch.

Force delivered to the jaw does not seem to be a good predictor of either HIC or rotational acceleration. It is uncertain, at this time, how force applied to the jaw is related to risk of MTBI or if the Hybrid III dummy in its current form has sufficient biofidelity to measure this parameter accurately. Hybrid III headforms with a deformable face insert such as that developed by Melvin et al, ${ }^{26}$ offer a state of the art test device. However, a headform with an articulating jaw may be needed to further explore head response to blows to the jaw region. While the peak jaw load represents only $26 \%$ of the impact force, it is consistent with the small region of the face instrumented with Tekscan.

\section{ACKNOWLEDGEMENTS}

The authors would like to acknowledge all the athletes who took time from their busy training schedules to participate in our study. We would also like to thank Capt. Wilson for allowing us to conduct our research in the boxing gym at Camp Lejeune, North Carolina, Dr Marilyn Boitano, and the staff of the United States Amateur Boxing Association for their commitment to safety. Special thanks are extended to Don Sherman whose involvement was essential for the success of the project.

\section{Authors' affiliations}

T J Walilko, D C Viano, C A Bir, Sports Biomechanics Laboratory, Department of BioMedical Engineering, Wayne State University, 818 W. Hancock, Detroit, MI 48201, USA

This study was funded by the United States Amateur Boxing Association and Wayne State University.

Consent was obtained for publication of figure 2

Competing interests: none declared

\section{REFERENCES}

1 Gartland S, Malik MHA, Lovell ME. Injury and injury rates in Muay Thai kick boxing. Br J Sports Med 2001;35:308-13.

2 Zazryn TR, Finch CF, McCrory P. A 16 year study of injuries to professional boxers in the state of Victoria, Australia. Br J Sports Med 2003;37:321-4.

3 Nakayama M. Dynamic karate. Palo Alto, CA: Kodansha International, 1966.

4 Walker JD. Karate strikes. Am J Physics 1975;43(10):845-9.

5 Smith PK, Hamill J. The effect of punching glove type and skill level on momentum transfer. J Hum Mov Stud 1986;12:153-61.

6 Atha J, Yeadon MR, Sandover J, et al. The damaging punch. Br Med J 1985;291(651 1):1756-7.

7 Smith MS, Dyson RJ, Hale T, et al. Development of a boxing dynamometer and its punch force discrimination efficacy. J Sports Sci 2000;18:445-50.

8 Joch W, Fritche P, Krause I. Biomechanical analysis of boxing. In: Morecki K, Fidelius K, Kdzior K, et al, eds. Biomechanics VII-A. Baltimore, MD: University Park Press, 1981:343-9.

9 Smith TA, Bishop PJ, Wells RP. Three dimensional analysis of linear and angular accelerations of the head experienced in boxing. In: Proceedings of the IRCOBI Conference on the Biomechanics of Impacts, Bergisch Gladbach (FRG). Bron, France: IRCOBI, 1988:271-85.

10 Padgaonkar AJ, Kreiger KW, King Al. Measurement of angular accelerations of a rigid body using linear accelerometers. J Appl Mech 1975;42:552-6.

11 Johnson J, Skorecki J, Wells RP. Peak acceleration of the head experienced in boxing. Med Biol Eng 1975; 13(3):396-403.

12 Unterharnscheidt F. About boxing: review of historical and medical aspects. Tex Rep Biol Med 1970;28:435-95.

13 Gadd CW. Use of a weighted-impulse criterion for estimating injury hazard. 8th Stapp Car Crash Conference. SAE paper no. 660793. Warrendale, PA: Society of Automotive Engineers, 1966.

14 Foster JK, Kortge JO, Wolanin MJ. Hybrid III - a biomechanically based crash dummy. 21 st Stapp Car Crash Conference. SAE paper no. 770938 Warrendale, PA: Society of Automotive Engineers, 1977.

15 Goldsmith W, Ommaya AK. In: Chapon BA, eds. Biomechanics of impact trauma. Head and neck injury criteria and tolerance levels. Amsterdam: Elsevier Science, 1984:149-90.

16 Gurdjian ES. Impact head injury. Springfield, IL: CC Thomas, 1975.

17 Ommaya AK, Goldsmith W, Thibault L. Biomechanics and neuropathology of adult and paediatric head injury: review. Br J Neurosurg 2002; 16(3):220-42.

18 Lissner HR. Experimental studies on the relationship between acceleration and intracranial pressure changes in man. Surg Gynecol Obstet 1960;111:329-38.

19 Gurdjian ES, Lissner HR, Patrick LM. Concussion mechanism and pathology In: 7th Stapp Car Crash Conference Proceedings. Springfield, IL: Charles C Thomas, 1965:470-82.

20 Prasad P, Mertz HJ. The position of the United States delegation to the ISO working group on the use of HIC in the automotive environment. SAE paper no. 851246 . Warrendale, PA: Society of Automotive Engineers, 1985.

21 Pellman EJ, Viano DC, Tucker AM, et al. Concussion in professional football: reconstruction of game impacts and injuries. Neurosurgery 2003;53:799-812.

22 Pellman EJ, Viano DC, Tucker AM, et al. Concussion in professional football: location and direction of helmet impacts - part 2. Neurosurgery 2003;53:1328-41.

23 Holbourn AHS. Mechanics of head injury. Lancet 1943;2:438-41.

24 Ommaya AK, Hirsch AE. Tolerances from cerebral concussions from head impact and whiplash in primates. J Biomech 1971;4:13-31.

25 Gennarelli T, Thibault L, Tomei G, et al. Directional dependence of axonal brain injury due to centroidal and non-centroidal acceleration, 31 st Stapp Car Crash Conference. SAE paper no. 872197. Warrendale, PA: Society of Automotive Engineers, 1987.

26 Melvin JW, Little WC, Smrcka J, et al. A biomechanic face for the Hybrid III dummy. 28th Stapp Car Crash Conference. SAE paper no. 952715. Warrendale, PA: Society of Automotive Engineers, 1995. 
27 Allsop DL, Warner CY, Wille MG et al. Facial impact response - a comparison of the Hybrid III dummy and human cadaver. SAE paper no. 881719. Warrendale, PA: Society of Automotive Engineers, 1988.

28 Newman JA, Gallup BM. Biofidelity improvements to the Hybrid III headform. 28th Stapp Car Crash Conference. SAE paper no. 841659. Warrendale, PA Society of Automotive Engineers, 1984.

29 SAE. Human mechanical impact response characteristics - responses of the human neck to inertial loading by the head for automotive seated postures. SAE paper no. J1460/2. Warrendale, PA: Society of Automotive Engineers, 1998.

30 SAE. Human tolerance to impact conditions as related to motor vehicle design. SAE paper no. J885. Warrendale, PA: Society of Automotive Engineers, 1986

31 Shee TR, Viano DC. Computing body segment trajectories in the Hybrid III dummy using linear accelerometer data. J Biomech Eng 1994;1 16(1):37-43.

32 Viano DC, Melvin JW, McCleary JD, et al. Measurement of head dynamics and facial contact forces in the Hybrid III dummy. 30th Stapp Car Crash Conference. SAE paper no. 861891. Warrendale, PA: Society of Automotive Engineers, 1986.
33 SAE. Instrumentation for impact test - part 1: electronic instrumentation SAE paper no. J211/1. Warrendale, PA: Society of Automotive Engineers, 1995.

34 SAE. Instrumentation for impact test - part 2: photographic instrumentation. SAE paper no. J211/2. Warrendale, PA: Society of Automotive Engineers, 2001.

35 Pincemaille Y, Trosseille X, Mack P. Some new data related to human tolerance obtained from volunteer boxers. SAE paper no. 892435. 33rd Stapp Car Crash Conference. Warrendale, PA: Society of Automotive Engineers, 1989.

36 Roberts A. Brain damage in boxers. London: Pitman Medical and Scientific Publishing, 1969

37 Unterharnscheidt F. A neurologist's reflections on boxing. I: Impact mechanics in boxing and injuries other than central nervous system damage. Rev Neurol 1995;23(121):661-74.

38 Clauser CE, McConville JT, Young JM. Weight, volume and center of mass of segments of the human body. Technical report AMRL-TR-69-70. Dayton, OH: Wright-Patterson Air Force Base, 1969.

\section{WHO SAID THAT?}

"Surprise me" (on where his wife should have him buried).

"You've got to be very careful if you don't know where you're going, because you might not get there".

"If you don't know where you are going, you will wind up somewhere else".

"If you can't imitate him, don't copy him".

"You better cut the pizza in four pieces because I'm not hungry enough to eat six".

"If you come to a fork in the road, take it".

"I made a wrong mistake".

"Do you mean now?" (when asked for the time).

"Baseball is $90 \%$ mental-the other half is physical".

"Slump? I ain't in no slump. I just ain't hittin".

"The game ain't over til it's over".

\section{Yogi Berra (1925- ), Baseballer}

awrence Peter "Yogi" Berra was born on May 12, 1925 in St. Louis, MO, USA. Yogi went on to become a fifteen time All Star, winning the American League MVP three times (1951, 1954, and 1955). He played in 14 World Series and holds numerous World Series records including most games by a catcher (63), hits (71), and times on a winning team (10), first in at bats, first in doubles, second in RBIs, third in home runs and BOBs.
After retiring as a player, Yogi went on to manage the NY Yankees, the NY Mets, and finally the Houston Astros until his retirement in 1992. Yogi is one of only a few managers to have won pennants in both the American and National Leagues. Yogi was elected the National Baseball Hall of Fame in 1972.

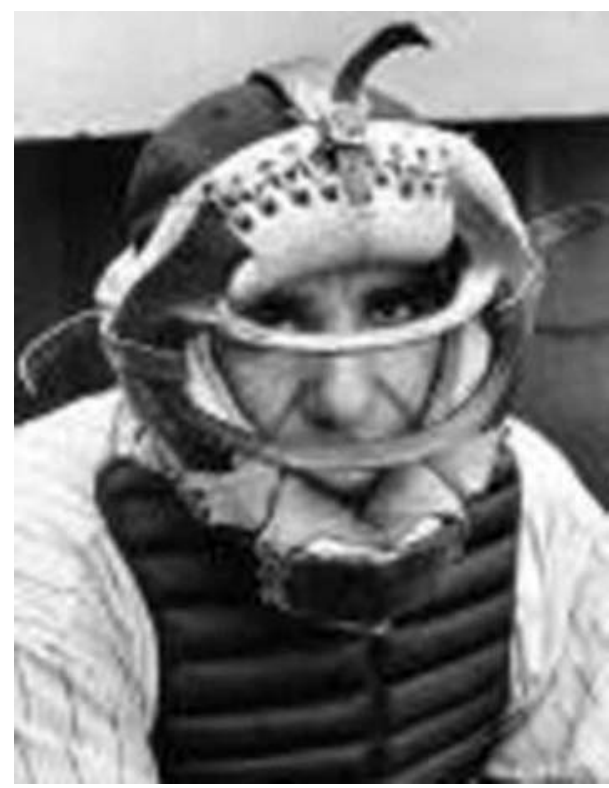

